\title{
Absorbed Radiation Doses to Staff After Implementation of a Radiopharmacy Clean Room
}

\author{
James A. Ponto, BCNP \\ Department of Radiology, University of Iowa Hospitals and Clinics, and College of Pharmacy, University of Iowa, \\ Iowa City, Iowa
}

In response to U.S. Pharmacopeia general chapter $<797>$ standards, a clean room was constructed for our in-house radiopharmacy. Previously, most patient doses were prepared as needed just before injection. Currently, to minimize repeated entries into the clean room, most patient doses are prepared in batches; that is, early morning and noontime preparation of doses to be injected at various times throughout the morning and the afternoon, respectively. Because these patient doses may be prepared well before injection time, radioactive decay necessitates higher amounts of radioactivity to be handled for patient dose preparation. Hence, absorbed radiation doses to staff, all of whom rotate into the radiopharmacy clean room in addition to their regular patient-related activities, were retrospectively evaluated. Methods: Monthly dosimetry reports for body (chest badge) and extremities (finger ring) were retrospectively reviewed for each staff member for 12 mo before and 12 mo after implementation of the radiopharmacy clean room. Monthly data were evaluated for average and SD, and 12-mo groups were evaluated using a paired $t$ test. Data for the second 12-mo period were also normalized to the same number of patient doses to account for an increase in procedure volume and were reevaluated. Results: Before the radiopharmacy clean room had been implemented, average monthly absorbed radiation doses to body and extremities were $23 \pm 15$ mrem $(0.23 \pm$ $0.15 \mathrm{mSv})$ and $93 \pm 59 \mathrm{mrem}(0.93 \pm 0.59 \mathrm{mSv})$, respectively. After the clean room had been implemented, average monthly absorbed radiation doses increased to $32 \pm 16$ mrem $(0.32 \pm$ $0.16 \mathrm{mSv})(P<0.001)$ and $121 \pm 89 \mathrm{mrem}(1.21 \pm 0.89 \mathrm{mSv})$ $(P=0.0015)$, respectively. When normalized for procedure volume, average monthly absorbed radiation doses after implementation of the clean room were still higher, at $29 \pm 15$ mrem $(0.29 \pm 0.15 \mathrm{mSv})(P=0.001)$ and $110 \pm 80 \mathrm{mrem}(1.10 \pm 0.80$ $\mathrm{mSv})(P=0.039)$, respectively. Conclusion: After implementation of a radiopharmacy clean room, absorbed radiation doses to body and extremities increased by $26 \%$ and $18 \%$, respectively, even after normalizing for procedure volume. Because absorbed radiation doses from other activities, such as patient dose administration and patient imaging, are assumed to remain relatively constant, these increases in absorbed radiation doses to staff are attributed to changes in work flow after implementation of the radiopharmacy clean room.

\footnotetext{
Received May 13, 2014; revision accepted Jul. 11, 2014

For correspondence or reprints contact: James A. Ponto, Nuclear Medicine, 3832 JPP, University of lowa Hospitals and Clinics, 200 Hawkins Dr., lowa City, IA 52242.

E-mail: james-ponto@uiowa.edu

Published online Aug. 28, 2014.

COPYRIGHT (C) 2014 by the Society of Nuclear Medicine and Molecular Imaging, Inc.
}

Key Words: radiation absorbed dose; radiopharmacy; clean room

J Nucl Med Technol 2014; 42:289-291

DOI: 10.2967/jnmt.114.143289

O ur institution has an in-house radiopharmacy lab, where ${ }^{99}$ Mo generators are eluted, reagent kits are radiolabeled, and patient doses are prepared. Traditionally, most patient doses were prepared (i.e., drawn up into a syringe) by staff technologists as needed just before injection. In response to the U.S. Pharmacopeia general chapter $<797>$ standards (1), our radiopharmacy lab was renovated to incorporate the applicable environmental conditions for compounding sterile preparations; that is, an International Organization for Standardization class 5 hood in a class 7 clean room with an adjacent class 8 anteroom. Entry into the clean room involves prerequisite hand cleansing and donning of shoe covers, a hair cover, a face mask, a gown, and gloves, a process that takes many minutes and incurs a cost of several dollars for disposable garb. To minimize repeated entries into the clean room, and thus the associated time and cost, most patient doses since implementation of the radiopharmacy clean room are prepared in batches; that is, early morning preparation of patient doses scheduled to be administered at various times throughout the morning, and noontime preparation of patient doses scheduled to be administered at various times throughout the afternoon. Because most of these patient doses are precalibrated for a future time, higher amounts of radioactivity are necessarily handled at the time of preparation to allow for radioactive decay. Hence, absorbed radiation doses to staff technologists who rotate into the radiopharmacy clean room are presumably higher after implementation of the radiopharmacy clean room than they were previously. The objective of this study was to retrospectively evaluate absorbed radiation doses to staff technologists before and after implementation of our radiopharmacy clean room.

\section{MATERIALS AND METHODS}

Anonymized monthly dosimeter reports for body (chest badge) and extremities (finger ring) were retrospectively reviewed for each staff technologist $(n=12)$ for the 12-mo period immediately 
TABLE 1

Summed Monthly Radiation Doses to the 12 Staff Technologists Before and After Implementation of Radiopharmacy Clean Room

\begin{tabular}{|c|c|c|c|c|c|c|}
\hline \multirow[b]{2}{*}{ Month } & \multicolumn{3}{|c|}{ Before } & \multicolumn{3}{|c|}{ After } \\
\hline & Body & Extremities & Doses $(n)$ & Body & Extremities & Doses $(n)$ \\
\hline July & $185(1.85)$ & $870(8.70)$ & 604 & 267 (2.67) & $1,090(10.90)$ & 546 \\
\hline August & $250(2.50)$ & $1,210(12.10)$ & 623 & 403 (4.03) & $1,510(15.10)$ & 700 \\
\hline September & 309 (3.09) & $1,240(12.40)$ & 694 & 362 (3.62) & $1,630(16.30)$ & 751 \\
\hline October & $261(2.61)$ & $1,050(10.50)$ & 613 & $413(4.13)$ & $1,720(17.20)$ & 703 \\
\hline November & 314 (3.14) & 1,330 (13.30) & 607 & 318 (3.18) & $1,300(13.00)$ & 718 \\
\hline December & $249(2.49)$ & $1,040(10.40)$ & 603 & $439(4.39)$ & $1,110(11.10)$ & 605 \\
\hline January & $236(2.36)$ & $1,020(10.20)$ & 608 & 417 (4.17) & $1,540(15.40)$ & 728 \\
\hline February & 317 (3.17) & $1,230(12.30)$ & 627 & 433 (4.33) & $1,680(16.80)$ & 737 \\
\hline March & 317 (3.17) & $1,240(12.40)$ & 701 & $426(4.26)$ & $1,700(17.00)$ & 846 \\
\hline April & 314 (3.14) & $880(8.80)$ & 672 & 387 (6.87) & $1,420(14.20)$ & 715 \\
\hline May & $250(2.50)$ & $1,070(10.70)$ & 647 & $375(3.75)$ & $1,520(15.20)$ & 729 \\
\hline June & 317 (3.17) & $1,190(11.90)$ & 655 & $322(3.22)$ & $1,260(12.60)$ & 654 \\
\hline Total & $3,319(33.19)$ & $13,370(133.70)$ & 7,654 & $4,562(45.62)$ & $17,480(174.80)$ & 8,432 \\
\hline
\end{tabular}

Data are mrem followed by $\mathrm{mSv}$ in parentheses.

before and the 12-mo period immediately after implementation of the radiopharmacy clean room. Individuals' absorbed radiation doses were summed for each month, and these summed data were evaluated for average and SD. Absorbed radiation doses for each of the 12-mo periods were evaluated for significant difference (i.e., $P<0.05$ ) using a paired $t$ test. Because of an increase in procedure volume, data for the second 12-mo period were also normalized for number of patient doses and reevaluated.

\section{RESULTS}

Data on summed absorbed radiation doses to staff technologists are presented in Table 1. The results of the evaluation of these absorbed radiation doses are presented in Table 2. Before implementation of the radiopharmacy clean room, average monthly absorbed radiation doses to body and extremities were $23 \pm 15 \mathrm{mrem}(0.23 \pm 0.15 \mathrm{mSv})$ and $93 \pm$ 59 mrem $(0.93 \pm 0.59 \mathrm{mSv})$, respectively. After implementation of the radiopharmacy clean room, average monthly absorbed radiation doses increased to $32 \pm 16$ mrem $(0.32 \pm$ $0.16 \mathrm{mSv})(P<0.001)$ and $121 \pm 89 \mathrm{mrem}(1.21 \pm 0.89$ $\mathrm{mSv})(P=0.0015)$, respectively. When normalized on the basis of the ratio of the number of patient doses for the 12-mo periods (i.e., 7,654 doses/8,432 doses), average monthly absorbed radiation doses to body and extremities after implementation of the radiopharmacy clean room were still higher, at $29 \pm 15 \mathrm{mrem}(0.29 \pm 0.15 \mathrm{mSv})(P=$ $0.001)$ and $110 \pm 80 \mathrm{mrem}(1.10 \pm 0.80 \mathrm{mSv})(P=0.039)$ respectively.

\section{DISCUSSION}

In our institution, staff technologists rotate through the radiopharmacy clean room, where they prepare patient doses, in addition to their traditional activities such as administering radiopharmaceuticals to patients and performing patient imaging procedures. Hence, only a fraction of their total absorbed radiation doses are from patient dose preparation in the radiopharmacy clean room. Because absorbed

\section{TABLE 2}

Average Monthly Radiation Doses to the 12 Staff Technologists Before and After Implementation of Radiopharmacy Clean Room

\begin{tabular}{|c|c|c|c|c|}
\hline \multirow[b]{2}{*}{ Interval } & \multicolumn{2}{|c|}{ Body } & \multicolumn{2}{|c|}{ Extremities } \\
\hline & Dose & $P^{*}$ & Dose & $P^{*}$ \\
\hline Before & $23 \pm 15(0.23 \pm 0.15)$ & & $93 \pm 59(0.93 \pm 0.59)$ & \\
\hline After & $32 \pm 16(0.32 \pm 0.16)$ & $<0.001$ & $121 \pm 89(1.21 \pm 0.89)$ & 0.0015 \\
\hline $\begin{array}{l}\text { After, normalized for no. of } \\
\text { patient doses }\end{array}$ & $29 \pm 15(0.29 \pm 0.15)$ & 0.001 & $110 \pm 80(1.10 \pm 0.80)$ & 0.039 \\
\hline
\end{tabular}

${ }^{\star}$ Paired $t$ test.

Data are mean mrem \pm SD followed by $\mathrm{mSv}$ in parentheses. 
radiation doses from their traditional activities are assumed to remain relatively constant, the increase in absorbed radiation doses after implementation of the radiopharmacy clean room can be attributed to changes in workflow, that is, handling higher amounts of radioactivity to prepare precalibrated patient doses.

Because there was an increase in procedure volume after clean room implementation, absorbed radiation doses for the second 12-mo period were normalized on the basis of the ratio of the numbers of patient doses from the 12-mo periods (i.e., 7,654 doses/8,432 doses). This simple normalization did not take into account any possible differences in procedure mix (e.g., type of procedure, adult vs. pediatric doses, or time of day). Normalization by procedure mix was beyond the scope of this study. However, any differences in procedure mix were judged to be minor and their influence on these analyses to be inconsequential.

\section{CONCLUSION}

After implementation of a radiopharmacy clean room, and the associated change in workflow, absorbed radiation doses to body and extremities of staff technologists increased significantly (by $26 \%$ and $18 \%$, respectively). However, such absorbed radiation doses remained well below regulatory limits, and the increased radiation risks are judged to be outweighed by the increased benefits (i.e., safety assurance) of aseptic patient dose preparation.

\section{DISCLOSURE}

No potential conflict of interest relevant to this article was reported.

\section{ACKNOWLEDGMENT}

This work was previously presented as a poster at the annual meeting of the Society of Nuclear Medicine and Molecular Imaging, St. Louis, MO, June 7-11, 2014.

\section{REFERENCE}

1. <797> Pharmaceutical Compounding: Sterile Preparations-USP 33/NF 28. Rockville, MD: United States Pharmacopeial Convention Inc.; 2010 . 